\title{
Thermal Image Super-Resolution Using Second-Order Channel Attention with Varying Receptive Fields
}

\author{
Nolan B. Gutierrez and William J. Beksi ^ \\ University of Texas at Arlington, Arlington TX, USA \\ nolan.gutierrez@mavs.uta.edu, william.beksi@uta.edu
}

\begin{abstract}
Thermal images model the long-infrared range of the electromagnetic spectrum and provide meaningful information even when there is no visible illumination. Yet, unlike imagery that represents radiation from the visible continuum, infrared images are inherently low-resolution due to hardware constraints. The restoration of thermal images is critical for applications that involve safety, search and rescue, and military operations. In this paper, we introduce a system to efficiently reconstruct thermal images. Specifically, we explore how to effectively attend to contrasting receptive fields (RFs) where increasing the RFs of a network can be computationally expensive. For this purpose, we introduce a deep attention to varying receptive fields network (AVRFN). We supply a gated convolutional layer with higher-order information extracted from disparate RFs, whereby an RF is parameterized by a dilation rate. In this way, the dilation rate can be tuned to use fewer parameters thus increasing the efficacy of AVRFN. Our experimental results show an improvement over the state of the art when compared against competing thermal image super-resolution (SR) methods.
\end{abstract}

Keywords: Thermal Imaging, Super-Resolution, Compression via Dilations

\section{Introduction}

The purpose of single image super-resolution (SISR) restoration is to determine the mapping between a possibly degraded low-resolution (LR) image and its high-resolution (HR) counterpart. Finding this arrangement is difficult due to the intractable nature of the problem. Techniques for discovering the mapping can be divided into two areas: interpolated and learning-based. Contemporary SISR has been dominated by deep learning which has demonstrated superiority over hand-crafted methods such as bicubic and bilinear interpolation.

\footnotetext{
* The authors acknowledge the Texas Advanced Computing Center (TACC) at the University of Texas at Austin for providing software, computational, and storage resources that have contributed to the research results reported within this paper.
} 
Convolutional neural networks (CNNs) have been shown to be successful at attending to visual images on tasks such as SISR. This includes squeeze-andexcitation methods for global excitation of feature maps [14,23], and the use of weight excitations [32,25]. Furthermore, ablation studies have conclusively shown that excitation networks for feature maps bring performance gains [40]. On another note, a variety of methods exist for modifying the RF of a CNN either through concatenation [22], deformation [10], or dilation [37]. In this work, we study how dilated convolutions offer compression through dilations. Concretely, we show how they modify the effective receptive fields (ERFs) of a CNN and how they interact with an existing enhancement known as second-order channel attention (SOCA) [11].

The development of infrared thermographic cameras has spurred researchers to carry out innovative research in the thermal image domain. Representing traditional SR, Mandanici et al. [28] combined geometric registration with projection and interpolation to produce HR thermal images. Naturally, researchers have recently investigated color-guided thermal image SR [8,2]. For example, a pyramidal network provided by Gupta et al. [12] attains accurate results by extracting edge-maps from RGB images at various levels of the network. Chudasama et al. [9] present an efficient SR network for thermal images by eliciting high-frequency details with a limited number of feature extraction modules. Other works have introduced popular loss functions to the field of thermal image SR $[1,17]$.

In this work we not only explore the use of efficient thermal SR, but we also provide complete benchmarks on three thermal imagery datasets. Furthermore, we compare the results of four architectural variants to assess performance gains or losses due to the compression of parameters in our SR network. In contrast to previous work on SISR, our proposed model applies SOCA to a concatenation of features produced from convolutions with changing RFs. An intermediate convolutional layer quantifies the importance of the values from each RF before passing this information to SOCA. The key contributions of our work are the following: (i) we show the effectiveness of SOCA for thermal image SR; (ii) we present a novel approach to sample from a foveated RF; (iii) we demonstrate an efficient network for multiple upscaling factors; (iv) we establish new benchmarks on public thermal image datasets. Our source code is available at [6].

The rest of this paper is organized as follows. We provide a summary of related thermal image SR work in Sec. 2, and the basics of SR in Sec. 3. In Sec. 4, we propose an architecture for transforming LR input images to super-resolved output images. In Sec. 5, we demonstrate our dilation-rate driven deep attention to varying receptive fields network through experimental results. Finally, we conclude in Sec. 6.

\section{Related Work}

Most recent developments in the image restoration domain focus on the visual image space $[31,39,4]$. The known importance of deeper CNNs in improving 
representational power has spurred the development of architectures that improve stability and provide better representations $[18,24]$. This is done not only through more residual connections [19,3,26], but also through structural preservation $[27,16]$, constrained hypotheses spaces [7], fast Fourier transform [33] and generative techniques [35], and student-teacher networks [21]. Additionally, SR works that focus on improving contextual information have employed different attention mechanisms [29] and enhanced inception modules [30].

\section{Preliminaries}

The task of super-resolving an LR image to its HR counterpart may be summarized by its image space and degradation model. Formally, the relationship can be defined as

$$
\boldsymbol{I}_{x}=D\left(\boldsymbol{I}_{y} ; \boldsymbol{\theta}\right),
$$

where $D$ (known as the degradation function) maps an HR image $\mathbf{I}_{y}$ to an LR image $\mathbf{I}_{x}$ with degradation parameters $\boldsymbol{\theta}$. Hence, SR can be reduced to finding the parameters of $D$, however it is an intractable process. Learning an SR model, $F$, can be formalized as

$$
\boldsymbol{I}_{y}=F\left(\boldsymbol{I}_{x} ; \boldsymbol{\theta}\right) .
$$

Furthermore, any optimization algorithm can be applied to find these parameters by minimizing an objective function,

$$
\hat{\boldsymbol{\theta}}=\underset{\boldsymbol{\theta}}{\arg \min } L\left(\hat{\boldsymbol{\theta}}_{y}, \boldsymbol{I}_{y}\right)+\lambda \psi(\boldsymbol{\theta}),
$$

where $\lambda$ is a small value that represents the importance of the regularization term $\psi$. This term may aid a model's ability to generalize to data never before seen. A common objective function to minimize is the mean squared error (MSE) otherwise known as the 2-norm,

$$
\operatorname{MSE}\left(\boldsymbol{I}_{x}, \boldsymbol{I}_{y}\right)=\frac{1}{N} \sum_{i=1}^{N}\left(\boldsymbol{I}_{x}(i)-\boldsymbol{I}_{y}(i)\right)^{2},
$$

where $N$ is the number of samples in a batch.

\section{Second-Order Channel Attention with Varying Receptive Fields}

We establish a novel deep learning architecture for thermal image SR as follows. First (Sec. 4.1), we provide a detailed description of SOCA. Second (Sec. 4.2), we expound upon how dilated convolutions are computed. Third (Sec. 4.3), we describe how CNNs can be compressed with respect to an ERF via compression through dilations. Fourth (Sec. 4.4), we incorporate residual in residual to aid in the stabilization of our network. Finally (Sec. 4.5), we apply SOCA to separate RFs (by dilation rates) within residual in residual to assist in the SR of LR images obtained with a bicubic degradation model. 


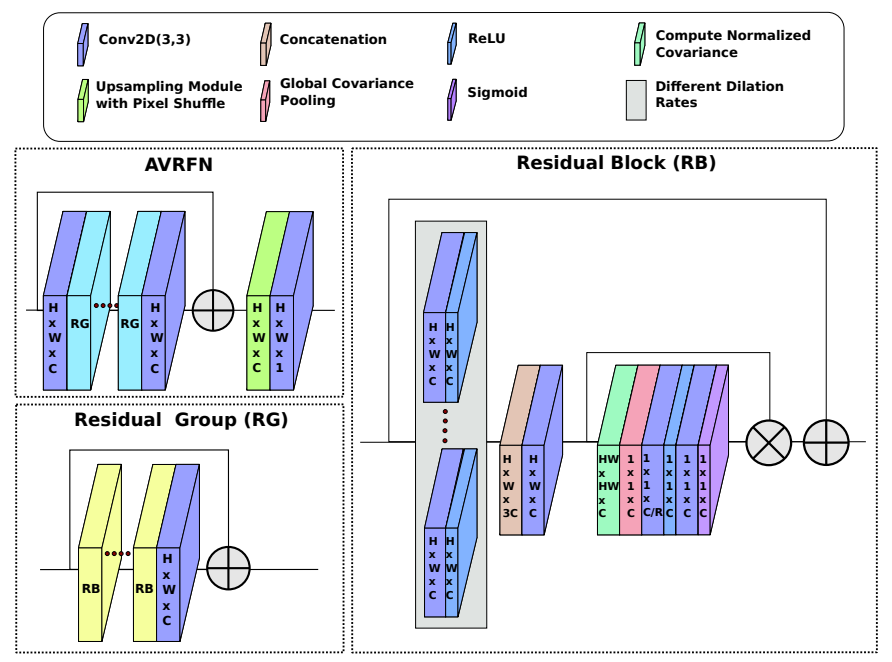

Fig. 1: An overview of our thermal imaging SR model.

\subsection{Second-Order Channel Attention}

We utilize an alternative SOCA network to enhance convolutional blocks by supplying a covariance matrix that allows for more discriminative representations. To produce these second-order statistics, the covariance normalization is obtained through Newton-Schulz iteration [13]. Additionally, this serves to speed up the computation. First, a feature map of dimension $H \times W \times C$ is reshaped into a feature map $\boldsymbol{X}$ of shape $H W \times C$. Second, the covariance matrix is calculated,

$$
\boldsymbol{\Sigma}=\boldsymbol{X} \boldsymbol{I}_{\boldsymbol{f}} \boldsymbol{X}^{\top}
$$

where $\boldsymbol{I}_{\boldsymbol{f}}=\frac{1}{s}\left(\boldsymbol{I}-\frac{1}{s} \mathbf{1}\right)$ and $s=H W . \boldsymbol{I}$ and $\mathbf{1}$ are the $m \times m$ identity matrix and the matrix of all ones, respectively. Then, the covariance matrix is prenormalized,

$$
\hat{\Sigma}=\frac{1}{\operatorname{tr}(\boldsymbol{\Sigma})} \boldsymbol{\Sigma},
$$

where $\operatorname{tr}(\cdot)$ denotes the matrix trace. Let $\boldsymbol{Y}_{0}=\hat{\boldsymbol{\Sigma}}$ and $\boldsymbol{Z}_{0}=\boldsymbol{I}$, then $\boldsymbol{Y}_{\boldsymbol{n}}$ and $\boldsymbol{Z}_{n}$ are obtained by

$$
\begin{aligned}
\boldsymbol{Y}_{n} & =\frac{1}{2} \boldsymbol{Y}_{n-1}\left(3 \boldsymbol{I}-\boldsymbol{Z}_{n-1} \boldsymbol{Y}_{n-1}\right), \\
\boldsymbol{Z}_{n} & =\frac{1}{2}\left(3 \boldsymbol{I}-\boldsymbol{Z}_{n-1} \boldsymbol{Y}_{n-1}\right) \boldsymbol{Z}_{n-1},
\end{aligned}
$$

with $\boldsymbol{Y}_{\boldsymbol{n}}$ and $\boldsymbol{Z}_{n}$ quadratically converging to $\boldsymbol{Y}$ and $\boldsymbol{Y}^{-1}$, respectively. The final normalized matrix after five iterations of Newton-Schulz is found by compensating the pre-normalization step with

$$
\hat{\boldsymbol{Y}}=\sqrt{\operatorname{tr}(\boldsymbol{\Sigma})} \boldsymbol{Y}_{N}
$$


Afterwards, global covariance pooling is applied to obtain a scalar-valued statistic $z_{i}$ for each channel $i$,

$$
\boldsymbol{z}_{i}=\frac{1}{C} \sum_{j}^{C} \hat{\boldsymbol{Y}}_{i j} .
$$

This permits the channel attention to capture correlations higher than the first order. In the next step, the sigmoid activation function serves as a gating mechanism that entrusts the network to selectively choose what to add to the incoming input features. To create this gating mechanism, we use two convolutional layers, $\boldsymbol{W}_{0}$ and $\boldsymbol{W}_{1}$, with rectified linear unit (ReLU) and sigmoid activation functions. Concretely,

$$
G(\boldsymbol{z})=\boldsymbol{W}_{1} *\left(\boldsymbol{W}_{0} * \boldsymbol{z}\right),
$$

where $*$ is the convolution operation and $G(\boldsymbol{z})$ is an attention map.

\subsection{Dilated Convolutions}

A dilated convolution multiplies a rate $l$ by $\Delta$ during the convolution operation, i.e.,

$$
\left(F *_{l} k\right)(\mathbf{p})=\sum_{\Delta \in \Omega_{r}} F(\mathbf{p}-l \cdot \Delta) k(\Delta),
$$

where $\Omega_{r}=[-r, r]^{2} \cap \mathbb{Z}^{2}, k: \Omega_{r} \rightarrow \mathbb{R}, \boldsymbol{p} \in \mathbb{Z}^{2}$ is a location on $\boldsymbol{X}, F: \mathbb{Z}^{2} \rightarrow \mathbb{R}$, and $*_{l}$ is an $l$-dilated convolution. In (12), $k$ is known as the kernel function which slides over $\boldsymbol{X}$. This allows a convolutional network to sample pixel values from a larger RF over the input features.

In the case of SR, it is advantageous to sample from different sized RFs depending on a number of factors including depth, resolution, and the scaling factor. We use dilated convolutions to extract features within each residual block. Specifically, the feature sets of three convolutional layers with varying dilation rates are concatenated and passed to an intermediate layer which pools the information from contrasting RFs. This intermediate layer effectively pools information at each feature map's location from a foveated RF where more parameters are concentrated towards the center of the field.

\subsection{Compression through Dilations}

We utilize dilated convolutions to artificially increase the ERFs of our CNN. An ERF is defined as the area containing any input pixel with a non-negligible impact on a particular output unit within a feature map [27]. In addition, we introduce the concept of compression through dilations as the case in which a CNN uses fewer parameters to increase an ERF with dilated convolutions compared to without dilated convolutions. For example, assuming we are using bias, two single-layer CNNs defined by Conv2D (Input_Shape $=(32,32,3), 64,(5,5))$ and Conv2D(Input_Shape $=(32,32,3), 64,(3,3)$, Dilation_Rate $=2)$ have an ERF area of 25 . However, our CNN has 160,064 and 57,664 parameters, respectively, giving a compression ratio of 2.776 . 


\subsection{Residual in Residual}

We stabilize our deep channel attention network by the addition of residual in residual (RIR) connections [40]. RIR entails two levels of connections with groups on the outer level and blocks on the inner level. More precisely,

$$
\hat{\mathbf{Y}}=\mathbf{X}+\mathbf{W} * R_{g}\left(R_{g-1}\left(\ldots R_{1}(\mathbf{X}) \ldots\right)\right) .
$$

$R_{g}$ is the $g$-th residual group and it is formulated as

$$
R_{g}(\mathbf{X})=\mathbf{X}+\mathbf{W} * B_{t}\left(B_{t-1}\left(\ldots B_{1}(\mathbf{X}) \ldots\right)\right),
$$

where $B_{t}$ is the $t$-th residual channel attention block. We apply SOCA to the features extracted from the convolutional layers with unique RFs. To obtain the individual RFs, we make the dilation rate of each convolutional layer exclusive and not equal to one for two of the layers. For example, if $\mathbf{W}_{\mathbf{1}}, \mathbf{W}_{\mathbf{2}}$, and $\mathbf{W}_{\mathbf{3}}$ are the weight sets associated with three convolutional layers, then the residual block is derived as

$$
B_{t}(\mathbf{X})=\mathbf{X}+\operatorname{SOCA}\left(\left[\mathbf{W}_{\mathbf{1}} * \mathbf{X}, \mathbf{W}_{\mathbf{2}} *_{2} \mathbf{X}, \mathbf{W}_{\mathbf{3}} *_{3} \mathbf{X}\right]\right)
$$

where $\left[\mathbf{W}_{\mathbf{1}}, \mathbf{W}_{\mathbf{2}}, \mathbf{W}_{\mathbf{3}}\right]$ constitutes the concatenation of $\mathbf{W}_{\mathbf{1}}, \mathbf{W}_{\mathbf{2}}$, and $\mathbf{W}_{\mathbf{3}}$ along the channel axis.

\subsection{Model Overview}

Our overall model is shown in Fig. 1. To upscale an input image, features are extracted from a series of residual groups and blocks within the RIR architecture similar to RCAN [40]. Pixel shuffle [36] is used to rearrange $\boldsymbol{X}$ of shape (H,W,C. $\left.r^{2}\right)$ to $(H \cdot r, W \cdot r, C)$ by periodically building a new feature map $P S(\boldsymbol{X})$ with pixel values from dissimilar channels according to the equation

$$
P S(\boldsymbol{X})_{x, y, c}=\boldsymbol{X}_{\lfloor x / r\rfloor,\lfloor y / r\rfloor, C \cdot r \cdot \bmod (y, r)+C \cdot \bmod (x, r)+c} .
$$

Finally, a single-channel convolutional layer reduces the number of channels to the same number as in the LR image.

\section{$5 \quad$ Experimental Results}

Our experiments model the downscaling of HR thermal images using a bicubic degradation model with statistical noise.

\subsection{Datasets}

We use the Thermal Image Super-Resolution (TISR) 2020 Challenge dataset, the FLIR Thermal Dataset for Algorithm Training (TDAT) [38], and the KAIST multispectral pedestrian detection benchmark dataset [15]. The TISR dataset 
consists of three sets of 1,021 images from three distinct cameras. These cameras include a Domo, Axis, and FLIR with a resolution of $(160 \times 120),(320 \times 240)$, and $(640 \times 480)$, respectively. Of these images, 60 were kept private, leaving 951 in the training set and 50 in the test set for each camera. For TDAT, we evaluate on only the first 100 images captured by a FLIR FC-6320 camera. Lastly, for the KAIST dataset, we collect every 200-th image from the day and night scenes and then evaluate on the set of 226 images. The images from the KAIST dataset were captured by a FLIR-A35 thermal camera with a resolution of $640 \times 480$.

The ground-truth dataset was created by first forming batches of 16 singlechannel image patches where each patch is of size scale $\times 48$. The LR images were then obtained by bicubicly interpolating these patches to a size of $48 \times 48$. For both training and testing, the images were preprocessed by adding Gaussian noise with a mean of 0 and a variance of 10 for the bicubic with noise degradation model. Finally, all elements of each LR patch are normalized and clipped to $[0,1]$.

\subsection{Implementation}

Our final architecture uses three residual groups with six residual blocks per group. Each convolutional layer has 64 filters resulting in a highly-efficient network. During training, the Adam optimizer [20], parameterized by a learning rate of $10^{-4}, \beta_{1}=0.9, \beta_{2}=0.999$, and $\epsilon=10^{-7}$, is applied to minimize the MSE of each batch for 300 epochs. Training with four NVIDIA GeForce GTX 1080 Ti GPUs took less than three hours per model.

\subsection{Evaluation}

For the experiments, we tested four variants of our architecture to evaluate the performance gains of the network. The variants are as follows.

- Dilated residual in residual (DDRR): SOCA in the residual block of Fig. 1 is replaced by a convolutional layer with a $3 \times 3$ kernel size and no activation function.

- Residual in residual with SOCA (RRSOCA): Our different dilation rate module in the residual block of Fig. 1 is replaced with a series of two convolutional layers each with a kernel size of $3 \times 3$ and a ReLU activation function.

- Compressed RCAN (CRCAN) via dilated convolutions: This architecture is similar to RRSOCA, but the first and second convolutional layers have a dilation rate of 1 and 2, respectively.

- Attention to varying receptive fields network (AVRFN): This is our proposed model as shown in Fig. 1.

Fig. 2 highlights example inputs, SR predictions, and the ground-truth images from the datasets. The added Gaussian noise produces heavily pixelated input images which presents very difficult conditions to evaluate our methods on. In all of our experiments, we use the peak signal-to-noise ratio (PSNR) and structural similarity index (SSIM) to evaluate each architectural variant. Table 1 shows the performance of our proposed AVRFN when evaluated on each 


\begin{tabular}{|c|c|c|c|c|}
\hline Test Set & Scale & Parameters & PSNR & SSIM \\
\hline AXIS Domo P1290 & & 2491009 & 30.097 & 0.846 \\
AXIS Q2901 & & 2491009 & 34.416 & 0.928 \\
FLIR FC-6320 & 2 & 2491009 & 37.116 & 0.942 \\
TDAT & & 2491009 & 31.184 & 0.733 \\
KAIST & & 2491009 & 40.612 & 0.962 \\
\hline AXIS Domo P1290 & & 2511809 & 26.831 & 0.747 \\
AXIS Q2901 & & 2511809 & 30.007 & 0.850 \\
FLIR FC-6320 & 3 & 251180 & 33.668 & 0.898 \\
TDAT & & 2511809 & 30.055 & 0.730 \\
KAIST & & 2511809 & 39.065 & 0.958 \\
\hline AXIS Domo P1290 & & 2507649 & 25.146 & 0.671 \\
AXIS Q2901 & & 2507649 & 27.724 & 0.784 \\
FLIR FC-6320 & 4 & 2507649 & 31.574 & 0.857 \\
TDAT & & 2507649 & 28.110 & 0.626 \\
KAIST & & 2507649 & 37.793 & 0.951 \\
\hline
\end{tabular}

Table 1: The results of our AVRFN model on images captured by the TISR [34], TDAT [38] and KAIST [15] datasets.

of the datasets. Additionally, Table 2 provides an ablation study which compares the performance of the various types of compression with different channel attention networks. Note that we were unable to make a fair comparison with related work found in [9] since the performance of our baseline method did not match. However, each of our variants performed better than the baseline RCAN architecture.

An interesting finding is that adding compression through dilations in the residual block of RCAN leads to improved performance. After each residual connection, the ERF resets to the kernel size of the succeeding CNN due to the easy pass-through of low-level information found in residual networks [5]. Contemporary work has found a correlation between larger ERFs and performance gains [5]. We hypothesize that by introducing compression through dilations in each residual block, we increase the ERF at a faster rate thus allowing for performance gains. An unexpected result is that DDRR, the only variant without any form of channel-attention, performs significantly worse. This confirms previous ablation studies [40], but it also shows that most of the performance gains arise from channel attention and not compression through dilations. In addition, our varying dilation rate module (AVRFN) improves performance over the baseline which shows that attending to different RFs can improve performance. Nonetheless, our highest performance gains are obtained when we simply add compression through dilations to the RCAN baseline.

\section{Conclusion}

In this work, we showed the advantage of attending to varying resolutions for the reconstruction of thermal images by efficiently parametrizing a convolutional 
layer with a dilation rate. Together with SOCA, our model achieves state-of-theart results on the task of thermal image SR and yields up-to-date benchmarks for the research community. In the future, we intend to look at ways in which training may be further stabilized and how attention to uncertainty maps can improve the computational efficiency of thermal image SR.

\begin{tabular}{|c|c|c|c|r|r|}
\hline Test Set & Model & Scale & Parameters & PSNR & SSIM \\
\hline \multirow{5}{*}{ AXIS Domo P1290 } & RRSOCA & 4 & 1661377 & 25.487 & 0.691 \\
& DDRR & 4 & 2839873 & 25.458 & 0.691 \\
& CRCAN & 4 & 2839873 & $\mathbf{2 5 . 4 9 1}$ & $\mathbf{0 . 6 9 2}$ \\
& RCAN & 4 & 1661377 & 25.239 & 0.682 \\
& AVRFN & 4 & 1917313 & 25.368 & 0.685 \\
\hline & RRSOCA & 4 & 1661377 & 28.167 & $\mathbf{0 . 8 0 2}$ \\
AXIS Q2901 & DDRR & 4 & 2839873 & 28.159 & 0.801 \\
& CRCAN & 4 & 2839873 & $\mathbf{2 8 . 1 8 9}$ & 0.802 \\
& RCAN & 4 & 1661377 & 27.923 & 0.795 \\
& AVRFN & 4 & 1917313 & 27.990 & 0.797 \\
\hline \multirow{5}{*}{ FLIR FC-6320 } & RRSOCA & 4 & 1661377 & 31.978 & 0.867 \\
& DDRR & 4 & 2839873 & 31.985 & 0.867 \\
& CRCAN & 4 & 2839873 & $\mathbf{3 2 . 0 0 2}$ & $\mathbf{0 . 8 6 7}$ \\
& RCAN & 4 & 1661377 & 31.756 & 0.861 \\
& AVRFN & 4 & 1917313 & 31.824 & 0.864 \\
\hline \multirow{5}{*}{ TDAT } & RRSOCA & 4 & 1661377 & 28.388 & 0.641 \\
& DDRR & 4 & 2839873 & $\mathbf{2 8 . 4 2 7}$ & $\mathbf{0 . 6 4 5}$ \\
& CRCAN & 4 & 2839873 & 28.426 & 0.645 \\
& RCAN & 4 & 1661377 & 28.271 & 0.636 \\
& AVRFN & 4 & 1917313 & 28.298 & 0.637 \\
\hline \multirow{5}{*}{ KAIST } & RRSOCA & 4 & 1661377 & $\mathbf{3 7 . 9 7 7}$ & $\mathbf{0 . 9 4 9}$ \\
& DDRR & 4 & 2839873 & 37.456 & 0.918 \\
& CRCAN & 4 & 2839873 & 37.573 & 0.922 \\
& RCAN & 4 & 1661377 & 37.089 & 0.938 \\
& AVRFN & 4 & 1917313 & 37.827 & 0.943 \\
\hline
\end{tabular}

Table 2: The results of our $\times 4$ model variants on images captured by the TISR [34], TDAT [38] and KAIST [15] datasets. 


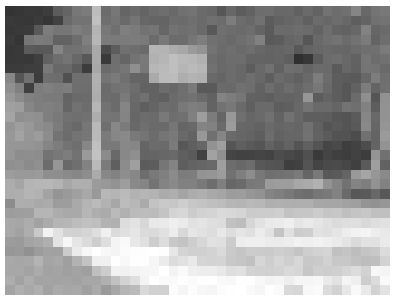

(a)

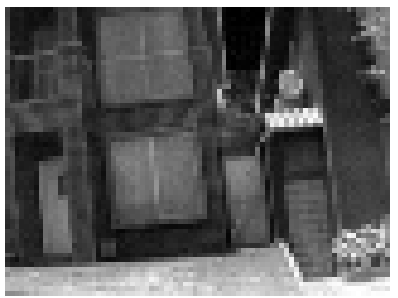

(d)

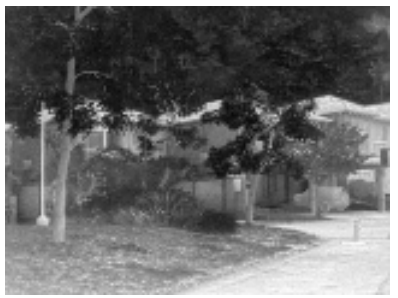

$(\mathrm{g})$

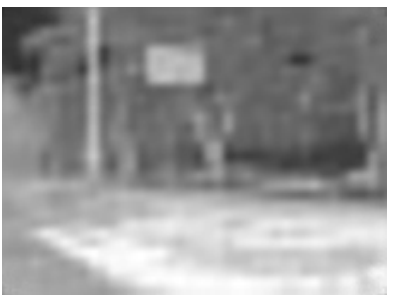

(b) $24.43 / 0.64$

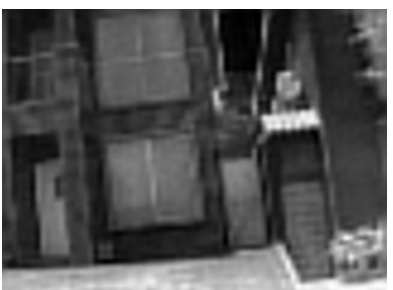

(e) $26.64 / 0.83$

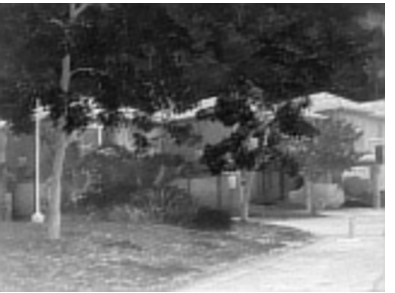

(h) $28.54 / 0.78$

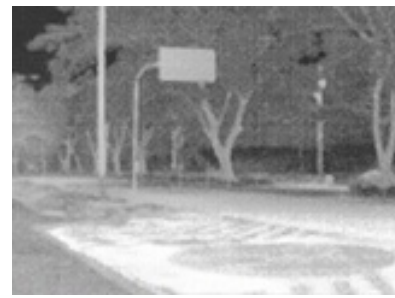

(c) GT

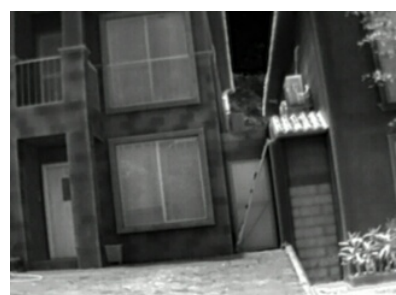

(f) $\mathrm{GT}$

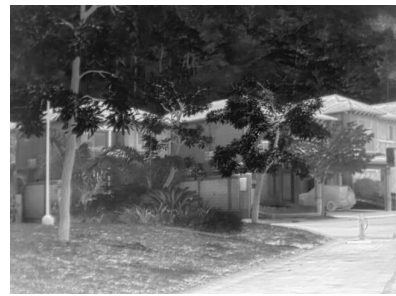

(i) GT

Fig. 2: Examples of (left column) downsampled images from (top row) lowresolution, (middle row) medium-resolution, and (bottom row) high-resolution thermal cameras, their $\times 4$ upscaled counterparts (middle column), and the ground truth (GT) (right column). Additionally, (b), (e), and (h) show the PSNR and SSIM scores, respectively, when evaluated against the ground truth. 


\section{References}

1. Almasri, F., Debeir, O.: Multimodal sensor fusion in single thermal image superresolution. In: Proceedings of the Asian Conference on Computer Vision. pp. 418433. Springer (2018)

2. Almasri, F., Debeir, O.: Rgb guided thermal super-resolution enhancement. In: Proceedings of the IEEE International Conference on Cloud Computing Technologies and Applications. pp. 1-5 (2018)

3. Anwar, S., Barnes, N.: Densely residual laplacian super-resolution. IEEE Transactions on Pattern Analysis and Machine Intelligence (2020)

4. Anwar, S., Khan, S., Barnes, N.: A deep journey into super-resolution: A survey. ACM Computing Surveys 53(3), 1-34 (2020)

5. Araujo, A., Norris, W., Sim, J.: Computing receptive fields of convolutional neural networks. Distill 4(11), e21 (2019)

6. https://github.com/robotic-vision-lab/Attention-With-VaryingReceptive-Fields-Network

7. Bahat, Y., Michaeli, T.: Explorable super resolution. In: Proceedings of the IEEE/CVF Conference on Computer Vision and Pattern Recognition. pp. 27162725 (2020)

8. Chen, X., Zhai, G., Wang, J., Hu, C., Chen, Y.: Color guided thermal image super resolution. In: Proceedings of the IEEE International Conference on Visual Communications and Image Processing. pp. 1-4 (2016)

9. Chudasama, V., Patel, H., Prajapati, K., Upla, K.P., Ramachandra, R., Raja, K., Busch, C.: Therisurnet - a computationally efficient thermal image super-resolution network. In: Proceedings of the IEEE/CVF Conference on Computer Vision and Pattern Recognition Workshops. pp. 86-87 (2020)

10. Dai, J., Qi, H., Xiong, Y., Li, Y., Zhang, G., Hu, H., Wei, Y.: Deformable convolutional networks. In: Proceedings of the IEEE International Conference on Computer Vision. pp. 764-773 (2017)

11. Dai, T., Cai, J., Zhang, Y., Xia, S.T., Zhang, L.: Second-order attention network for single image super-resolution. In: Proceedings of the IEEE International Conference on Computer Vision. pp. 11065-11074 (2019)

12. Gupta, H., Mitra, K.: Pyramidal edge-maps based guided thermal super-resolution. arXiv preprint arXiv:2003.06216 (2020)

13. Higham, N.J.: Functions of Matrices: Theory and Computation. SIAM (2008)

14. Hu, J., Shen, L., Sun, G.: Squeeze-and-excitation networks. In: Proceedings of the IEEE Conference on Computer Vision and Pattern Recognition. pp. 7132-7141 (2018)

15. Hwang, S., Park, J., Kim, N., Choi, Y., So Kweon, I.: Multispectral pedestrian detection: Benchmark dataset and baseline. In: Proceedings of the IEEE Conference on Computer Vision and Pattern Recognition. pp. 1037-1045 (2015)

16. Isobe, T., Jia, X., Gu, S., Li, S., Wang, S., Tian, Q.: Video super-resolution with recurrent structure-detail network. In: Proceedings of the European Conference on Computer Vision. pp. 645-660. Springer (2020)

17. Kansal, P., Nathan, S.: A multi-level supervision model: A novel approach for thermal image super resolution. In: Proceedings of the IEEE/CVF Conference on Computer Vision and Pattern Recognition Workshops. pp. 94-95 (2020)

18. Kim, J., Kwon Lee, J., Mu Lee, K.: Accurate image super-resolution using very deep convolutional networks. In: Proceedings of the IEEE Conference on Computer Vision and Pattern Recognition. pp. 1646-1654 (2016) 
19. Kim, J., Kwon Lee, J., Mu Lee, K.: Deeply-recursive convolutional network for image super-resolution. In: Proceedings of the IEEE Conference on Computer Vision and Pattern Recognition. pp. 1637-1645 (2016)

20. Kingma, D.P., Ba, J.: Adam: A method for stochastic optimization. arXiv preprint arXiv:1412.6980 (2014)

21. Lee, W., Lee, J., Kim, D., Ham, B.: Learning with privileged information for efficient image super-resolution. In: Proceedings of the European Conference on Computer Vision. pp. 465-482. Springer (2020)

22. Li, J., Fang, F., Mei, K., Zhang, G.: Multi-scale residual network for image superresolution. In: Proceedings of the European Conference on Computer Vision. pp. 517-532 (2018)

23. Li, T., Wu, B., Yang, Y., Fan, Y., Zhang, Y., Liu, W.: Compressing convolutional neural networks via factorized convolutional filters. In: Proceedings of the IEEE/CVF Conference on Computer Vision and Pattern Recognition. pp. 39773986 (2019)

24. Lim, B., Son, S., Kim, H., Nah, S., Mu Lee, K.: Enhanced deep residual networks for single image super-resolution. In: Proceedings of the IEEE/CVF Conference on Computer Vision and Pattern Recognition Workshops. pp. 136-144 (2017)

25. Lin, X., Ma, L., Liu, W., Chang, S.F.: Context-gated convolution. In: Proceedings of the European Conference on Computer Vision. pp. 701-718. Springer (2020)

26. Liu, J., Zhang, W., Tang, Y., Tang, J., Wu, G.: Residual feature aggregation network for image super-resolution. In: Proceedings of the IEEE/CVF Conference on Computer Vision and Pattern Recognition. pp. 2359-2368 (2020)

27. Luo, W., Li, Y., Urtasun, R., Zemel, R.: Understanding the effective receptive field in deep convolutional neural networks. In: Proceedings of the International Conference on Neural Information Processing Systems. pp. 4905-4913 (2016)

28. Mandanici, E., Tavasci, L., Corsini, F., Gandolfi, S.: A multi-image super-resolution algorithm applied to thermal imagery. Applied Geomatics 11(3), 215-228 (2019)

29. Mei, Y., Fan, Y., Zhou, Y., Huang, L., Huang, T.S., Shi, H.: Image super-resolution with cross-scale non-local attention and exhaustive self-exemplars mining. In: Proceedings of the IEEE/CVF Conference on Computer Vision and Pattern Recognition. pp. 5690-5699 (2020)

30. Muhammad, W., Aramvith, S.: Multi-scale inception based super-resolution using deep learning approach. Electronics 8(8), 892 (2019)

31. Nasrollahi, K., Moeslund, T.B.: Super-resolution: a comprehensive survey. Machine Vision and Applications 25(6), 1423-1468 (2014)

32. Quader, N., Bhuiyan, M.M.I., Lu, J., Dai, P., Li, W.: Weight excitation: builtin attention mechanisms in convolutional neural networks. In: Proceedings of the European Conference on Computer Vision. pp. 87-103. Springer (2020)

33. Reddy, B.S., Chatterji, B.N.: An fft-based technique for translation, rotation, and scale-invariant image registration. IEEE Transactions on Image Processing 5(8), 1266-1271 (1996)

34. Rivadeneira, R.E., Sappa, A.D., Vintimilla, B.X., Guo, L., Hou, J., Mehri, A., Behjati Ardakani, P., Patel, H., Chudasama, V., Prajapati, K., Upla, K.P., Ramachandra, R., Raja, K., Busch, C., Almasri, F., Debeir, O., Nathan, S., Kansal, P., Gutierrez, N., Mojra, B., Beksi, W.J.: Thermal image super-resolution challengepbvs 2020. In: Proceedings of the IEEE/CVF Conference on Computer Vision and Pattern Recognition Workshops. pp. 96-97 (2020)

35. Saharia, C., Ho, J., Chan, W., Salimans, T., Fleet, D.J., Norouzi, M.: Image superresolution via iterative refinement. arXiv preprint arXiv:2104.07636 (2021) 
36. Shi, W., Caballero, J., Huszár, F., Totz, J., Aitken, A.P., Bishop, R., Rueckert, D., Wang, Z.: Real-time single image and video super-resolution using an efficient sub-pixel convolutional neural network. In: Proceedings of the IEEE Conference on Computer Vision and Pattern Recognition. pp. 1874-1883 (2016)

37. Szegedy, C., Liu, W., Jia, Y., Sermanet, P., Reed, S., Anguelov, D., Erhan, D., Vanhoucke, V., Rabinovich, A.: Going deeper with convolutions. In: Proceedings of the IEEE Conference on Computer Vision and Pattern Recognition. pp. 1-9 (2015)

38. Teledyne FLIR LLC: Free FLIR Thermal Dataset for Algorithm Training (2021), https://www.flir.in/oem/adas/adas-dataset-form/

39. Wang, Z., Chen, J., Hoi, S.C.: Deep learning for image super-resolution: A survey. IEEE Transactions on Pattern Analysis and Machine Intelligence (2020)

40. Zhang, Y., Li, K., Li, K., Wang, L., Zhong, B., Fu, Y.: Image super-resolution using very deep residual channel attention networks. In: Proceedings of the European Conference on Computer Vision. pp. 286-301 (2018) 